\title{
SIMULATION OF TRANSIENT ULTRASONIC WAVE PROPAGATION IN FLUID-LOADED HETEROGENEOUS CORTICAL BONE
}

\author{
Vu Hieu Nguyen, Salah Naili \\ Université Paris-Est, France
}

\begin{abstract}
This work deals with the ultrasonic wave propagation in the cortical layer of long bones which is known as being a functionally-graded anisotropic material coupled with fluids. The motivation arises from mechanical modeling of the ultrasound axial transmission technique in vivo for cortical long bone which is known as being a functionally-graded anisotropic material. The proposed method is based on a combined Laplace-Fourier transform which substitutes a problem defined by partial differential equations into a system of differential equations established in the frequency-wavenumber domain. In the spectral domain, as radiation conditions may be exactly introduced in the infinite fluid halfspaces, only the heterogeneous solid layer needs to be analyzed using finite element method. Several numerical tests are presented showing very good performance of the proposed approach.
\end{abstract}

Keywords: Spectral finite element, transient wave, ultrasound, anisotropic, vibroacoustic, cortical bone, axial transmission.

\section{INTRODUCTION}

In recent years, quantitative ultrasound (QUS) has demonstrated its promising potential in assessment of in vivo bone characteristics. An advantage of QUS over X-ray techniques is its ability to give some information about the elastic properties and defects of bones. Moreover, ultrasound is non-ionizing and the ultrasonic apparatus is relatively inexpensive and can be made portable.

For measuring in vivo properties of cortical long bones, a so-called "axial transmission" (AT) technique has been developed [1]. The axial transmission technique uses a set of ultrasonic transducers (transmitters and receivers) placed on a line in contact with the skin along the bone axial axis. The transmitter emits an ultrasound pulse wave (around $250 \mathrm{KHz}-2 \mathrm{MHz}$ ) that propagates along the cortical layer of bones. The analysis of the signals received at the receivers can allow to the quantification of the geometrical information as well as mechanical characteristics of the cortical bone at the measured skeletal site $[2,3]$. Mechanical modeling of this experiment deals with considering a model describing vibro-acoustic interactions of a solid waveguide (which represents the cortical bone) coupled with two fluid media (which represents soft tissues such as skin or marrow). The cortical bone may be described as plate-like or cylindrical-like structures. This technique of nondestructive testing used to evaluate the material properties requires careful analysis of 
the reflections, conversion modes and interferences of longitudinal and shear waves within the bone structure.

Many studies have focused on the modeling of guided waves in long bones by using fluid-loaded (multilayer) plate models. The analysis of wave phenomena in the structure have been considered in the frequency-domain $[4,5,6,7]$ or in the time-domain $[8,9,10,11]$. For the analysis of multilayer structures in the time-domain, there are mainly two approaches. The first one involves using (semi-)analytical methods such as the generalized ray/Cagniard-de-Hoop technique [9] or the direct stiffness matrix method [12]. The second one involves using numerical methods such as the finite difference method (FDM) $[8,13,14]$ or the finite element method (FEM) $[11,13,15]$. Although the analytical methods are attractive to obtain reliable transient responses of the structure, numerical methods are often more efficient to treat problems with inhomogeneous materials or complex geometries. However, most numerical methods require important computational costs, especially for problems in the high-frequency domain. Moreover, absorbing boundary conditions are required when considering unbounded domains $[13,16,17]$.

For analyzing the waveguide with geometrical and mechanical properties which are constant along one or two directions, an efficient approach called the Hybrid Numerical Method (HNM, see e.g. [18, 19, 20]), alternatively called Spectral Finite Element Method (SFEM, see e.g. [21, 22]). The key point of this method consists of using a hybrid algorithm which begins by employing the Fourier transform (with respect to time and to the longitudinal direction of the waveguide) to transform problem into the frequency-wavenumber domain. Then, the wave equations in the spectral domain governed in a cross-section (or even a 1D domain in the case of infinite plates or axisymmetric waveguides), which may actually have inhomogeneous material properties, can be easily handled using the finite element method $[10,18,19,22]$. In comparison with classical FEM which requires extensive discretization due to small wavelengths for high frequency problems, the SFEM presents important advantages in time and memory saving. However, noting that when using the SFEM, finite element analysis should be performed at each value in the set of frequencies/wavenumbers, the computational cost increases rapidly with the size of the cross-sectional domain. In addition, when performing the inverse Fourier transform to obtain the time response of undamped structures, some numerical difficulties may arise because of singular-valued solutions at the poles of the frequency response solution. In order to improve the numerical stability, an appropriate artificial damping would be added into the model, removing the singularity or almost singular behavior near the real axis of the complex frequency response function [21].

In this work, we propose to use the spectral finite element method to study time response solve the transient wave propagation problem in a layered or functionally graded elastic plate loaded by two infinite fluid domains. This geometrical configuration is appropriate to study the radio-frequency signals obtained from the AT technique in order to recover the geometrical and mechanical characteristics of cortical bone. By assuming that the fluids are homogeneous, the general solutions of acoustic waves in fluids can be solved analytically in the spectral domain, so only the solid domain needs to be analyzed by finite element method. 
The paper is organized as follows. In Section 2, we present a description of the geometrical configuration of the coupled fluid-solid system. Then, the governing equations as well as the boundary and interface conditions of the considered problem are given. Section 3 presents the problem formulation in the Laplace-Fourier domain (i.e. frequencywavenumber domain). Section 4 provides the spectral finite element formulation and the procedure to obtain the solution in the spatio-temporal domain. Section 5 presents some numerical results. It begins with several validations which are performed in comparison with finite element results. Then, we show a case in which the influence of the variation of mechanical properties in the solid layer is considered. Finally, the conclusion and perspectives of this work are given in Section 6.

\section{STATEMENT OF THE PROBLEM}

In this section, we describe an idealized model of the AT technique performed on cortical bone. The modeling consists of a plate of infinite extent (representing the cortical bone layer) and two semi-infinite ideal fluid media (representing soft tissues) (see Fig. 1). A source of pressure and a array of the receivers are placed in the upper fluid medium. The excitation represents an infinite line source perpendicular to the considered plane. As a consequence, a two-dimensional (2D) model is appropriate to modeling wave phenomena in the system.

\subsection{Description of the problem}

Let $\mathbf{R}\left(\mathrm{O} ; \mathbf{e}_{1}, \mathbf{e}_{2}\right)$ be the reference Cartesian frame where $\mathrm{O}$ is the origin of the space and $\left(\mathbf{e}_{1}, \mathbf{e}_{2}\right)$ is an orthonormal basis for this space. The coordinates of a point $\boldsymbol{x}$ in $\mathbf{R}$ are specified by $\left(x_{1}, x_{2}\right)$ and the time is denoted by $t$.

Consider an infinite solid layer occupying the domain $\Omega^{b}$ that represents the cortical bone plate with a constant thickness $h\left(\Omega^{b}=\left\{\boldsymbol{x}\left(x_{1}, x_{2}\right) ; 0 \geq x_{2} \leq-h\right\}\right)$. This bone plate is loaded on its upper and lower surfaces by two fluid halfspaces. The upper fluid domain is denoted by $\Omega_{1}^{f}\left(\Omega_{1}^{f}=\left\{\boldsymbol{x}\left(x_{1}, x_{2}\right) ; x_{2} \geq 0\right\}\right)$ and the lower one is denoted by $\Omega_{2}^{f}$ $\left(\Omega_{2}^{f}=\left\{\boldsymbol{x}\left(x_{1}, x_{2}\right) ; x_{2} \leq-h\right\}\right)$. The interfaces between the bone $\left(\Omega^{b}\right)$ and the fluids $\left(\Omega_{1}^{f}\right.$ and $\Omega_{2}^{f}$ ) are denoted by $\Gamma_{1}^{b f}$ and $\Gamma_{2}^{b f}$, respectively (see Fig. 1).

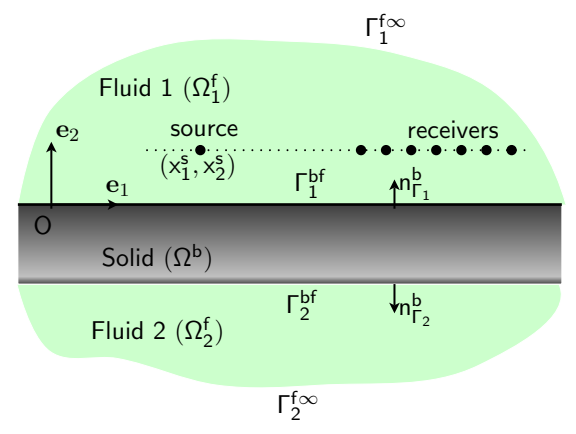

Fig. 1. Geometrical configuration of the trilayer model for ultrasound axial transmission test 
Both fluid domains $\left(\Omega_{1}^{f}\right.$ and $\left.\Omega_{2}^{f}\right)$ are considered as homogeneous fluid media and the solid plate is assumed to be an anisotropic elastic solid. Moreover, according to the cortical bone properties, we assume that mechanical properties of the solid may vary along the $\boldsymbol{e}_{2}$-axis but they are homogeneous along the longitudinal direction described by the $\boldsymbol{e}_{1}$-axis.

The system is excited by an acoustic source located at a point $\boldsymbol{x}^{s}=\left(x_{1}^{s}, x_{2}^{s}\right)$ located in the upper fluid domain $\Omega_{1}^{f}$.

\subsection{Governing equations}

The governing equations for the wave propagation in a coupled fluid-solid system can be found in many references, see e.g. [23]. Here, we only outline the main equations to solve the considered trilayer problem. In what follows, we denote respectively by $\nabla \cdot, \nabla$ and $\nabla^{2}$ the divergence, gradient and Laplacian operators with respect to $\boldsymbol{x}$ and by the superposed dot, differentiation with respect to time $t$.

Governing equations in the fluid domains $\left(\Omega_{1}^{f}\right.$ and $\left.\Omega_{2}^{f}\right)$. Let us first consider the fluid domain $\Omega_{1}^{f}$. Since the considered compressible fluid is assumed to be inviscid, the equation of motion in the domain $\Omega_{1}^{f}$ can be described by using a scalar field $p_{1}$, which satisfies the wave equation:

$$
\frac{1}{c_{1}^{2}} \ddot{p}_{1}-\nabla^{2} p_{1}=\dot{Q}, \quad \forall \boldsymbol{x} \in \Omega_{1}^{f}
$$

where $p_{1}(\boldsymbol{x}, t)$ is the acoustic pressure in the domain $\Omega_{1}^{f}, Q(\boldsymbol{x}, t)$ is the acoustic source density, the constant $c_{1}$ is the wave velocity in $\Omega_{1}^{f}$ at rest which depends on the bulk modulus $K_{1}$ of the fluid and its mass density $\rho_{1}$ by the relation $c_{1}=\sqrt{K_{1} / \rho_{1}}$.

The impulsive infinite line source acting at $\boldsymbol{x}^{s}=\left(0, x_{2}^{s}\right)$ is described as:

$$
\dot{Q}=\rho_{1} F(t) \delta\left(x_{1}\right) \delta\left(x_{2}-x_{2}^{s}\right),
$$

where $F(t)$ is a given real scalar function depending only on the time and $\delta($.$) is Dirac's$ delta function.

Similarly to the equation of motion in the domain $\Omega_{1}^{f}$, the pressure field $p_{2}$ in the fluid domain $\Omega_{2}^{f}$ reads:

$$
\frac{1}{c_{2}^{2}} \ddot{p}_{2}-\nabla^{2} p_{2}=0, \quad \forall \boldsymbol{x} \in \Omega_{2}^{f},
$$

where $c_{2}=\sqrt{K_{2} / \rho_{2}}$ is the wave velocity in $\Omega_{2}^{f}, K_{2}$ and $\rho_{2}$ are respectively the bulk modulus of the fluid and its mass density at rest.

Governing equations in the solid domain $\left(\Omega^{b}\right)$. We denote by $\boldsymbol{u}(\boldsymbol{x}, t)=$ $\left\{u_{1}, u_{2}\right\}^{T}$ the time-dependent vector of displacement at a point $\boldsymbol{x}$ located in the solid domain $\Omega^{b}$, where the superscript "T" designates the transpose operator. The associated linearized strain and stress tensors are denoted by $\boldsymbol{\epsilon}(\boldsymbol{u})$ and $\boldsymbol{\sigma}(\boldsymbol{u})$, respectively. Neglecting the body force, the equation of motion in the solid domain $\Omega^{b}$ is given by:

$$
\rho \ddot{\boldsymbol{u}}-\nabla \cdot \boldsymbol{\sigma}=\mathbf{0}, \quad \forall \boldsymbol{x} \in \Omega^{b}
$$

where $\rho$ denotes the mass density of the solid. 
For the finite element development afterwards, it would be more convenient to rewrite the stress and strain tensors in vectorial form: $\mathbf{s}=\left\{\sigma_{11}, \sigma_{22}, \sigma_{12}\right\}^{T}$ and $\mathbf{e}=$ $\left\{\epsilon_{11}, \epsilon_{22}, 2 \epsilon_{12}\right\}^{T}$. Using this notation, the strain-displacement relation is given by:

$$
\mathbf{e}=\mathbb{L} \boldsymbol{u}, \quad \mathbb{L}=\left[\begin{array}{cc}
\frac{\partial}{\partial x_{1}} & 0 \\
0 & \frac{\partial}{\partial x_{2}} \\
\frac{\partial}{\partial x_{2}} & \frac{\partial}{\partial x_{1}}
\end{array}\right],
$$

and the constitutive equation describing the anisotropic elastic behavior of the solid reads:

$$
\mathbf{s}=\mathbf{C e}, \quad \text { with } \mathbf{C}=\left[\begin{array}{lll}
c_{11} & c_{12} & c_{16} \\
c_{12} & c_{22} & c_{26} \\
c_{16} & c_{26} & c_{66}
\end{array}\right]
$$

where $\mathbf{C}$ is the elasticity tensor written in the Voigt matrix notation. The equation of the motion (4) may be restated as follows:

$$
\rho \ddot{\boldsymbol{u}}-\mathbb{L}^{T} \mathbf{s}=\mathbf{0},
$$

which becomes in substituting the constitutive equation (6):

$$
\rho \ddot{\boldsymbol{u}}-\mathbb{L}^{T} \mathbf{C L} \boldsymbol{u}=\mathbf{0} .
$$

We recall that the material properties of the solid depend only on $x_{2}$, i.e. $\rho=\rho\left(x_{2}\right)$ and $\mathbf{C}=\mathbf{C}\left(x_{2}\right)$.

Boundary and interface conditions. The interface conditions at the fluid-solid interfaces $\left(\Gamma_{1}^{b f}\right.$ and $\left.\Gamma_{2}^{b f}\right)$ may be described by the same way. The condition required for the continuity of normal velocities at the interfaces reads:

$$
\nabla p_{\alpha} \cdot \boldsymbol{n}_{\Gamma_{\alpha}}^{f}=-\rho_{\alpha} \ddot{\boldsymbol{u}}^{\Gamma_{\alpha}} \cdot \boldsymbol{n}_{\Gamma_{\alpha}}^{f}, \quad \forall \boldsymbol{x} \in \Gamma_{\alpha}^{b f} \quad(\alpha=1,2)
$$

whereas the condition of the continuity of normal stresses at the interfaces requires:

$$
\boldsymbol{\sigma} \boldsymbol{n}_{\Gamma_{\alpha}}^{b}=-p_{\alpha} \boldsymbol{n}_{\Gamma_{\alpha}}^{b}, \quad \forall \boldsymbol{x} \in \Gamma_{\alpha}^{b f} \quad(\alpha=1,2)
$$

where $\boldsymbol{n}_{\Gamma_{\alpha}}^{f}(\alpha=1,2)$ is the unit normal vector at the interface $\Gamma_{\alpha}^{b f}$ pointing out of $\Omega_{\alpha}^{f}$ and $\boldsymbol{n}_{\Gamma_{\alpha}}^{b}(\alpha=1,2)$ is the unit normal vector of the interface $\Gamma_{\alpha}^{b f}$ pointing out of $\Omega^{b}$ (see Fig. $1)$.

Finally, the radiation conditions of two fluid halfspaces in the far field reads:

$$
p_{\alpha} \rightarrow 0, \quad \forall \boldsymbol{x} \rightarrow \Gamma_{\alpha}^{f \infty}(\alpha=1,2) .
$$

Boundary value problem in terms of $\left(p_{1}, \boldsymbol{u}, p_{2}\right)$. This paragraph rewrites all previous equations in a more compact form. Note that for the considered configuration (see Fig. 1) wherein $\boldsymbol{n}_{\Gamma_{1}}^{b}=-\boldsymbol{n}_{\Gamma_{1}}^{f}=\{0,1\}^{T}$ and $\boldsymbol{n}_{\Gamma_{2}}^{b}=-\boldsymbol{n}_{\Gamma_{2}}^{f}=\{0,-1\}^{T}$, the equations in terms of $\left(p_{1}, \boldsymbol{u}, p_{2}\right)$ of the coupled fluid-solid problem may be represented as follows: 
○ Fluid domain $\Omega_{1}^{f}$ :

$$
\begin{aligned}
& \frac{1}{c_{1}^{2}} \ddot{p}_{1}-\nabla^{2} p_{1}=\rho_{1} F(t) \delta\left(x_{1}\right) \delta\left(x_{2}-x_{2}^{s}\right), \quad \forall \boldsymbol{x} \in \Omega_{1}^{f}, \\
& \frac{1}{\rho_{1}} \frac{\partial p_{1}}{\partial x_{2}}=-\ddot{u}_{2}, \quad \forall \boldsymbol{x} \in \Gamma_{1}^{b f}, \\
& p_{1} \rightarrow 0, \quad \forall \boldsymbol{x} \rightarrow \Gamma_{1}^{f \infty} .
\end{aligned}
$$

o Solid domain $\Omega^{b}$ :

$$
\begin{aligned}
& \rho \ddot{\boldsymbol{u}}-\mathbb{L}^{T} \mathbf{C} \mathbb{L} \boldsymbol{u}=\mathbf{0}, \quad \forall \boldsymbol{x} \in \Omega^{b}, \\
& \mathbf{t}=\left\{0,-p_{1}\right\}^{T}, \quad \forall \boldsymbol{x} \in \Gamma_{1}^{b f}, \\
& \mathbf{t}=\left\{0,-p_{2}\right\}^{T}, \quad \forall \boldsymbol{x} \in \Gamma_{2}^{b f},
\end{aligned}
$$

where $\mathbf{t}:=\left\{\sigma_{12}, \sigma_{22}\right\}^{T}$.

$\circ$ Fluid domain $\Omega_{2}^{f}$ :

$$
\begin{aligned}
& \frac{1}{c_{2}^{2}} \ddot{p}_{2}-\nabla^{2} p_{2}=0, \quad \forall \boldsymbol{x} \in \Omega_{2}^{f}, \\
& \frac{1}{\rho_{2}} \frac{\partial p_{2}}{\partial x_{2}}=-\ddot{u}_{2}, \quad \forall \boldsymbol{x} \in \Gamma_{2}^{b f}, \\
& p_{2} \rightarrow 0, \quad \forall \boldsymbol{x} \rightarrow \Gamma_{2}^{f \infty} .
\end{aligned}
$$

\section{EQUATIONS IN THE LAPLACE-FOURIER DOMAIN}

The problem presented in (12) - (20) deals with solving a system of linear partial differential equations in which the coefficients are homogeneous in the longitudinal direction given by $x_{1}$-axis. Here, we propose to solve the system as follows: (i) the system of equations is firstly transformed into frequency-wavenumber domain by using a Fourier transform with respect to $x_{1}$ combined with a Laplace transform with respect to $t$; (ii) in the frequency-wavenumber domain, the equations in both fluid domains are solved analytically giving impedance boundary and interface conditions for the solid layer that may be solved by the finite element method; (iii) the space-time solution is finally obtained by performing two inverse transforms.

The general form of a Laplace-Fourier transform (LF) applied to a real-valued function $y\left(x_{1}, x_{2}, t\right)$ denoted by $\tilde{y}\left(k_{1}, x_{2}, s\right)$ is defined as:

$$
\begin{aligned}
\tilde{y}\left(k_{1}, x_{2}, s\right): & =\mathcal{L}\left\{\mathcal{F}\left[y\left(x_{1}, x_{2}, t\right)\right]\right\} \\
& =\int_{0}^{\infty}\left(\int_{-\infty}^{+\infty} y\left(x_{1}, x_{2}, t\right) e^{-i k_{1} x_{1}} d x_{1}\right) e^{-s t} d t
\end{aligned}
$$

where $i=\sqrt{-1}, s \in \mathbb{C}$ is the complex Laplace variable, $k_{1} \in \mathbb{R}$ is the real Fourier variable representing the wavenumber on the $x_{1}$-axis, $\mathbb{R}$ and $\mathbb{C}$ denote the set of all the real and complex numbers, respectively. 
In $\left(s-k_{1}\right)$ domain, the time derivative and the spatial derivative with respect to $x_{1}$ can be replaced by $\frac{\partial(.)}{\partial t} \rightarrow-s($.$) and \frac{\partial(.)}{\partial x_{1}} \rightarrow i k_{1}($.$) , respectively.$

\subsection{Transformed problem in $\left(s-k_{1}\right)$ domain for the fluids $\Omega_{1}^{f}$ and $\Omega_{2}^{f}$}

By applying the Laplace-Fourier transform (21) to Eqs. (12) - (14), the problem so defined is reduced to be a boundary-value differential equation for $\tilde{p}_{1}$ with respect only to $x_{2}$ :

$$
\begin{aligned}
& \left(\frac{s^{2}}{c_{1}^{2}}+k_{1}^{2}\right) \tilde{p}_{1}-\frac{\partial^{2} \tilde{p}_{1}}{\partial x_{2}^{2}}=\rho_{1} \tilde{F}_{0}(s) \delta\left(x_{2}-x_{2}^{s}\right), \\
& \frac{1}{\rho_{1}}\left(\frac{\partial \tilde{p}_{1}}{\partial x_{2}}\right)_{x_{2}=0}=-s^{2} \tilde{u}_{2}\left(k_{1}, 0, s\right), \\
& \tilde{p}_{1} \rightarrow 0, \quad \forall x_{2} \rightarrow+\infty
\end{aligned}
$$

of which the solution for $\tilde{p}_{1}$ may be expressed in semi-explicit form as:

$$
\begin{aligned}
& \tilde{p}_{1}=-\frac{1}{2} \frac{\rho_{1}}{\alpha_{1}} \tilde{F}_{0}\left(e^{-\alpha_{1}\left(x_{2}^{s}-x_{2}\right)}+e^{-\alpha_{1}\left(x_{2}^{s}+x_{2}\right)}\right)+\frac{\rho_{1}}{\alpha_{1}} s^{2} \tilde{U}_{21} e^{-\alpha_{1} x_{2}}, \text { for } 0 \leq x_{2} \leq x_{2}^{s}, \\
& \tilde{p}_{1}=-\frac{1}{2} \frac{\rho_{1}}{\alpha_{1}} \tilde{F}_{0}\left(e^{\alpha_{1}\left(x_{2}^{s}-x_{2}\right)}+e^{-\alpha_{1}\left(x_{2}^{s}+x_{2}\right)}\right)+\frac{\rho_{1}}{\alpha_{1}} s^{2} \tilde{U}_{21} e^{-\alpha_{1} x_{2}}, \text { for } x_{2} \geq x_{2}^{s},
\end{aligned}
$$

where $\alpha_{1}:=\sqrt{\frac{s^{2}}{c_{1}^{2}}+k_{1}^{2}}, \tilde{U}_{21}$ is the LF transform of the vertical displacement of the solid layer at the upper fluid-solid interface $\Gamma_{1}^{b f}$ which is defined by $\tilde{U}_{21}:=\tilde{u}_{2}\left(k_{1}, 0, s\right)$.

Similarly, applying the LF-transform to Eqs. (18) - (20), the general solution of $\tilde{p}_{2}$ may be obtained:

$$
\tilde{p}_{2}=-\frac{\rho_{2}}{\alpha_{2}} s^{2} \tilde{U}_{22} e^{\alpha_{2}\left(x_{2}+h\right)}, \quad \forall x_{2} \leq-h,
$$

where $\alpha_{2}=\sqrt{\frac{s^{2}}{c_{2}^{2}}+k_{1}^{2}}$ and $\tilde{U}_{22}:=\tilde{u}_{2}\left(k_{1},-h, s\right)$.

\subsection{Transformed problem in $\left(s-k_{1}\right)$ domain for the solid $\left(\Omega^{b}\right)$}

By applying the LF-transform (21) to the system of equations associated with the solid given by Eqs. (15) - (17), we obtain:

$$
\rho s^{2} \tilde{\boldsymbol{u}}-\tilde{\mathbb{L}}^{T} \mathbf{C} \tilde{\mathbb{L}} \tilde{\boldsymbol{u}}=\mathbf{0},
$$

where

$$
\begin{aligned}
& \tilde{\mathbf{u}}=\left(\begin{array}{l}
\tilde{u}_{1} \\
\tilde{u}_{2}
\end{array}\right), \quad \tilde{\mathbb{L}}=i k_{1} \mathbf{L}_{1}+\mathbf{L}_{2} \frac{\partial}{\partial x_{2}} \\
& \text { with } \quad \mathbf{L}_{1}=\left[\begin{array}{ll}
1 & 0 \\
0 & 0 \\
0 & 1
\end{array}\right] \text { and } \mathbf{L}_{2}=\left[\begin{array}{ll}
0 & 0 \\
0 & 1 \\
1 & 0
\end{array}\right]
\end{aligned}
$$


For each value of the couple $\left(s, k_{1}\right) \in \mathbb{C} \times \mathbb{R}$, the differential equations given by Eq. (28) lead to a system for $\tilde{\boldsymbol{u}}$ with respect only to $x_{2}$ :

$$
\left(s^{2} \mathbf{A}_{1}+k_{1}^{2} \mathbf{A}_{2}\right) \tilde{\mathbf{u}}-i k_{1}\left(\mathbf{A}_{3}+\mathbf{A}_{3}^{T}\right) \frac{\partial \tilde{\mathbf{u}}}{\partial x_{2}}-\mathbf{A}_{4} \frac{\partial^{2} \tilde{\mathbf{u}}}{\partial x_{2}^{2}}=\mathbf{0}
$$

where the matrices $\mathbf{A}_{1}, \mathbf{A}_{2}, \mathbf{A}_{3}$ and $\mathbf{A}_{4}$, which depend only on the physical parameters, are defined by:

$$
\begin{aligned}
& \mathbf{A}_{1}=\left[\begin{array}{ll}
\rho & 0 \\
0 & \rho
\end{array}\right] ; \quad \mathbf{A}_{2}=\mathbf{L}_{1}^{T} \mathbf{C} \mathbf{L}_{1}=\left[\begin{array}{ll}
c_{11} & c_{16} \\
c_{16} & c_{66}
\end{array}\right] ; \\
& \mathbf{A}_{3}=\mathbf{L}_{2}^{T} \mathbf{C L}_{1}=\left[\begin{array}{ll}
c_{16} & c_{66} \\
c_{12} & c_{26}
\end{array}\right] ; \quad \mathbf{A}_{4}=\mathbf{L}_{2}^{T} \mathbf{C L}_{2}=\left[\begin{array}{ll}
c_{66} & c_{26} \\
c_{26} & c_{22}
\end{array}\right] .
\end{aligned}
$$

The interface conditions given by Eqs. (16) - (17) in $\left(s-k_{1}\right)$ domain read:

$$
\tilde{\mathbf{t}}(0)=\left(\begin{array}{c}
0 \\
-\tilde{p}_{1}(0)
\end{array}\right), \quad \tilde{\mathbf{t}}(-h)=\left(\begin{array}{c}
0 \\
-\tilde{p}_{2}(-h)
\end{array}\right)
$$

in which the solution for $\tilde{p}_{1}(0)$ at $\Gamma_{1}^{b f}$ and the solution for $\tilde{p}_{2}(-h)$ at $\Gamma_{2}^{b f}$ can be determined by using Eqs. (26) and (27), respectively:

$$
\begin{aligned}
& \tilde{p}_{1}(0)=-\frac{\rho_{1}}{\alpha_{1}}\left(\tilde{F}_{0} e^{-\alpha_{1} x_{2}^{s}}-s^{2} \tilde{U}_{21}\right), \\
& \tilde{p}_{2}(-h)=-\frac{\rho_{2}}{\alpha_{2}} s^{2} \tilde{U}_{22} .
\end{aligned}
$$

Thus, the interface conditions given by Eq. (34) can be rewritten as:

$$
\tilde{\mathbf{t}}(0)=\mathbf{F}_{0}-\mathbf{P}_{1} \tilde{\mathbf{u}}(0), \quad \tilde{\mathbf{t}}(-h)=\mathbf{P}_{2} \tilde{\mathbf{u}}(-h),
$$

where

$$
\begin{aligned}
& \mathbf{F}_{0}=\left(\begin{array}{c}
0 \\
\frac{\rho_{1}}{\alpha_{1}} \tilde{F}_{0} e^{-\alpha_{1} x_{2}^{s}}
\end{array}\right), \\
& \mathbf{P}_{1}=\left[\begin{array}{cc}
0 & 0 \\
0 & \frac{\rho_{1}}{\alpha_{1}} s^{2}
\end{array}\right], \quad \mathbf{P}_{2}=\left[\begin{array}{cc}
0 & 0 \\
0 & \frac{\rho_{2}}{\alpha_{2}} s^{2}
\end{array}\right] .
\end{aligned}
$$

Moreover, it would be useful to note that the constitutive relation of the solid given by Eq. (6) in the $\left(s k_{1}\right)$ domain may be restated as:

$$
\tilde{\mathbf{s}}=\mathbf{C} \tilde{\mathbb{L}} \tilde{\mathbf{u}}
$$

and the vector $\tilde{\mathbf{t}}$ (which is given by $\tilde{\mathbf{t}}=\mathbf{L}_{2}^{T} \tilde{\mathbf{s}}$ ) can be expressed by:

$$
\tilde{\mathbf{t}}=i k_{1} \mathbf{A}_{3} \tilde{\mathbf{u}}+\mathbf{A}_{4} \frac{\partial \tilde{\mathbf{u}}}{\partial x_{2}} \text {. }
$$




\section{SPECTRAL FINITE ELEMENT FORMULATION AND TIME-SPACE SOLUTION}

\subsection{Weak formulation in the solid domain}

The weak formulation of the boundary-value problem given by Eqs. (31) - (37) may be now introduced using the classic procedure (see e.g. [15]). Let $\mathrm{e}^{a d}$ be the function space which comprises all sufficiently smooth complex-valued admissible functions: $x_{2} \in$ ]$-h, 0\left[\rightarrow \delta \tilde{\mathbf{u}}\left(x_{2}\right) \in \mathbb{C} \times \mathbb{C}\right.$. The conjugate transpose of $\delta \tilde{\mathbf{u}}$ is denoted by $\delta \tilde{\mathbf{u}}^{*}$.

Upon integrating the Eq. (31) against a test function $\delta \tilde{\mathbf{u}}^{*} \in \mathfrak{C}^{a d}$ and integrating by parts, we obtain:

$$
\begin{aligned}
\int_{-h}^{0} \delta \tilde{\mathbf{u}}^{*} & \left(s^{2} \mathbf{A}_{1}+k_{1}^{2} \mathbf{A}_{2}-i k_{1} \mathbf{A}_{3}^{T} \frac{\partial}{\partial x_{2}}\right) \tilde{\mathbf{u}} d x_{2}+\int_{-h}^{0} \frac{\partial \delta \tilde{\mathbf{u}}^{*}}{\partial x_{2}}\left(i k_{1} \mathbf{A}_{3}+\mathbf{A}_{4} \frac{\partial}{\partial x_{2}}\right) \tilde{\mathbf{u}} d x_{2} \\
- & {\left[\delta \tilde{\mathbf{u}}^{*}\left(i k_{1} \mathbf{A}_{3}+\mathbf{A}_{4} \frac{\partial}{\partial x_{2}}\right) \tilde{\mathbf{u}}\right]_{-h}^{0}=0 }
\end{aligned}
$$

The last terms associated with boundary conditions given by Eq. (42) may be calculated by using the relations (37) and (41):

$$
\begin{aligned}
{\left[\delta \tilde{\mathbf{u}}^{*}\left(i k_{1} \mathbf{A}_{3}+\mathbf{A}_{4} \frac{\partial}{\partial x_{2}}\right) \tilde{\mathbf{u}}\right]_{-h}^{0} } & =\left[\delta \tilde{\mathbf{u}}^{*} \tilde{\mathbf{t}}\right]_{-h}^{0} \\
& =\delta \tilde{\mathbf{u}}^{*}(0) \mathbf{F}_{0}-\delta \tilde{\mathbf{u}}^{*}(0) \mathbf{P}_{1} \tilde{\mathbf{u}}(0)-\delta \tilde{\mathbf{u}}^{*}(-h) \mathbf{P}_{2} \tilde{\mathbf{u}}(-h)
\end{aligned}
$$

The weak formulation of the differential equation (31) reads: For all $k_{1}$ fixed in $\mathbb{R}$ and for all $s$ fixed in $\mathbb{C}$, find $\tilde{\mathbf{u}}\left(k_{1}, x_{2}, s\right) \in \mathcal{C}^{a d}$ such that:

$$
\begin{aligned}
& \int_{-h}^{0} \delta \tilde{\mathbf{u}}^{*}\left(s^{2} \mathbf{A}_{1}+k_{1}^{2} \mathbf{A}_{2}-i k_{1} \mathbf{A}_{3}^{T} \frac{\partial}{\partial x_{2}}\right) \tilde{\mathbf{u}} d x_{2}+\int_{-h}^{0} \frac{\partial \delta \tilde{\mathbf{u}}^{*}}{\partial x_{2}}\left(i k_{1} \mathbf{A}_{3}+\mathbf{A}_{4} \frac{\partial}{\partial x_{2}}\right) \tilde{\mathbf{u}} d x_{2} \\
& \quad+\delta \tilde{\mathbf{u}}^{*}(0) \mathbf{P}_{1} \tilde{\mathbf{u}}(0)+\delta \tilde{\mathbf{u}}^{*}(0) \mathbf{P}_{2} \tilde{\mathbf{u}}(-h)=\delta \tilde{\mathbf{u}}^{*}(0) \mathbf{F}_{0}, \quad \forall \delta \tilde{\mathbf{u}} \in \mathcal{C}^{a d}
\end{aligned}
$$

\subsection{Finite element formulation}

We proceed by introducing a finite element mesh of the domain $[-h, 0]$ which contains $n^{e l}$ elements $\Omega_{e}:[-h, 0]=\bigcup_{e} \Omega_{e}\left(e=1, \ldots, n^{e l}\right)$. By the Galerkin finite element method, both functions $\tilde{\mathbf{u}}$ and $\delta \tilde{\mathbf{u}}$ in each element $\Omega_{e}$ are approximated using the same shape function:

$$
\tilde{\mathbf{u}}\left(x_{2}\right)=\mathbf{N}_{e} \mathbf{U}_{e}, \quad \delta \tilde{\mathbf{u}}\left(x_{2}\right)=\mathbf{N}_{e} \delta \mathbf{U}_{e}, \quad \forall x_{2} \in \Omega_{e},
$$

where $\mathbf{N}_{e}$ is the shape function, $\mathbf{U}_{e}$ and $\delta \mathbf{U}_{e}$ are the vectors of nodal solutions of $\tilde{\mathbf{u}}$ and $\delta \tilde{\mathbf{u}}$ within the element $\Omega_{e}$, respectively. Substituting (45) into (44) and assembling the elementary matrices, we obtain a linear system of equations:

$$
\left(\mathbf{K}+\mathbf{K}^{\Gamma}\right) \mathbf{U}=\mathbf{F},
$$

where $\mathbf{U}$ is the global nodal displacement vector, $\mathbf{K}$ is the global "stiffness matrix" of the solid, $\mathbf{K}^{\Gamma}$ represents the operator of coupling between the fluids and the solid and $\mathbf{F}$ is the external force vector. For all couples $\left(s, k_{1}\right)$ fixed in $\mathbb{C} \times \mathbb{R}$, these quantities may be 
expressed by:

$$
\begin{aligned}
\mathbf{K} & =s^{2} \mathbf{K}_{A_{1}}+k_{1}^{2} \mathbf{K}_{A_{2}}+i k_{1} \mathbf{K}_{A_{3}}+\mathbf{K}_{A_{4}}, \\
\mathbf{K}^{\Gamma} & =\operatorname{Diag}\left\{0, \frac{\rho_{1}}{\alpha_{1}} s^{2}, 0, \ldots, 0, \frac{\rho_{2}}{\alpha_{2}} s^{2}\right\}, \\
\mathbf{F} & =\left\{0, \frac{\rho_{1}}{\alpha_{1}} \tilde{F}_{0} e^{-\alpha_{1} x_{2}^{s}}, 0, \ldots, 0\right\}^{T},
\end{aligned}
$$

where the matrices $\mathbf{K}_{A_{1}}, \mathbf{K}_{A_{2}}, \mathbf{K}_{A_{3}}$ and $\mathbf{K}_{A_{4}}$ are independent of $s$ and $k_{1}$ and are defined by:

$$
\begin{aligned}
& \mathbf{K}_{A_{1}}=\bigcup_{e} \int_{\Omega_{e}} \mathbf{N}_{e}^{T} \mathbf{A}_{1} \mathbf{N}_{e} d x_{2}, \quad \mathbf{K}_{A_{2}}=\bigcup_{e} \int_{\Omega_{e}} \mathbf{N}_{e}^{T} \mathbf{A}_{2} \mathbf{N}_{e} d x_{2}, \\
& \mathbf{K}_{A_{3}}=\bigcup_{e} \int_{\Omega_{e}}\left\{\mathbf{N}_{e}^{\prime T} \mathbf{A}_{3} \mathbf{N}_{e}\right\}_{a} d x_{2}, \quad \mathbf{K}_{A_{4}}=\bigcup_{e} \int_{\Omega_{e}} \mathbf{N}_{e}^{\prime T} \mathbf{A}_{4} \mathbf{N}_{e}^{\prime} d x_{2},
\end{aligned}
$$

in which the notation $\{.\}_{a}$ is devoted for the skew part of the $\{$.$\} and \{.\}^{\prime}$ means the differentiation with respect to $x_{2}$.

Remark 1. We recall that the solid layer is assumed to be heterogeneous and the elementary matrices $\mathbf{A}_{1}, \mathbf{A}_{2}, \mathbf{A}_{3}$ and $\mathbf{A}_{4}$ (see Eqs. (32) - (33)) are not constant but vary with respect to $x_{2}$. Here, the elementary matrices (see Eqs. (50) - (51)) are numerically computed by using the Gauss quadrature technique. However, when the material properties of the solid layer are homogeneous, analytical expressions of elementary matrices may be determined.

Remark 2. To reduce the computational cost, the matrices $\mathbf{K}_{A_{1}}, \mathbf{K}_{A_{2}}, \mathbf{K}_{A_{3}}$ and $\mathbf{K}_{A_{4}}$ have only to be computed once before performing a loop on $s$ and $k_{1}$. For each value of $s$ and $k_{1}$, the global matrix $\mathbf{K}$ is obtained by performing the summation (see Eq. (47)). Moreover, one may note that:

$$
\mathbf{K}\left(-k_{1}, s\right)=\mathbf{K}^{T}\left(k_{1}, s\right) .
$$

\subsection{Computation of time-space solution}

For fixed values of $\left(s, k_{1}\right)$ in the Laplace-Fourier transformed domain, the solution of $\tilde{\mathbf{u}}$ may be computed by solving the system of linear equations in the complex domain (46). The solutions for $\tilde{p}_{1}$ and $\tilde{p}_{2}$ in two fluid domains may be then determined by using equations (25) and (26), respectively. In order to obtain the spatio-temporal solution, we need to perform a numerical inverse Laplace-Fourier transform.

In this paper, the inverse Fourier transform is computed by using the usual FFT (Fast Fourier Transform) technique. The inverse Laplace transform is carried out using the Quadrature Convolution Method which has been shown to be a very efficient technique for computing the time response solution in many dynamic problems [24].

Let $N_{1}$ and $\Delta k_{1}$ respectively the sampling number and sampling rate of the wavenumber $k_{1}$ using for the FFT procedure. The space-solution is reconstructed upon on a broad 
spectral band $\left[-k_{1}^{\max }, k_{1}^{\max }\right]$ where $k_{1}^{\max }=N_{1} \Delta k_{1} / 2$. The space interval is then calculated by $\Delta x_{1}=2 \pi /\left(N_{1} \Delta k_{1}\right)$ corresponding to a space span $\left[-x_{1}^{\max }, x_{1}^{\max }\right]$ with $x_{1}^{\max }=\pi / \Delta k_{1}$.

Using the Convolution Quadrature Method (CQM), the solution in the time domain, e.g. of $p_{1}(\boldsymbol{x}, t)$, corresponding to a time-dependent excitation function $f_{0}(t)$ is evaluated by a convolution integral:

$$
\begin{aligned}
p_{1}(\boldsymbol{x}, t) & =\int_{0}^{t} \pi_{1}(\boldsymbol{x}, \tau) f_{0}(t-\tau) d \tau \\
& =\int_{0}^{t} \mathcal{L}^{-1}\left[\tilde{\pi}_{1}(\boldsymbol{x}, s)\right] f_{0}(t-\tau) d \tau,
\end{aligned}
$$

where $\pi_{1}$ is the fundamental solution that corresponds to a Dirac distribution excitation (i.e. the Green function) and $\tilde{\pi}_{1}$ is its Laplace transform. The CQM supplies a stable quadrature formula to approximate this convolution integral in the time-domain and the required computational time with this formula is attractive:

$$
p_{1}(\boldsymbol{x}, n \Delta t)=\sum_{m=0}^{n} \omega_{n-m}(\boldsymbol{x}, \Delta t) f_{0}(m \Delta t), \quad\left(n=0,1, \ldots, N_{t}\right),
$$

where $\Delta t$ is the time step defined by $\Delta t=T / N_{t}$ and $T$ being the duration of the phenomenon studied.

The weights $\omega_{n}(\boldsymbol{x}, \Delta t)$ are determined in terms of $\tilde{\pi}_{1}(\boldsymbol{x}, s)$ :

$$
\omega_{n}(\boldsymbol{x}, \Delta t)=\frac{R^{-n}}{N_{t}} \sum_{\ell=0}^{N_{t}-1} \tilde{\pi}_{1}\left(\boldsymbol{x}, \frac{1}{\Delta t} \gamma\left(R e^{i \ell \frac{2 \pi}{N_{t}}}\right)\right) e^{-i n \ell \frac{2 \pi}{N_{t}}},
$$

where $\gamma(z)=3 / 2-2 z+z^{2} / 2$ and $R$ is the radius of a circle in the domain of analyticity of $\tilde{\pi}_{1}$. Choosing $R^{N_{t}}=\sqrt{\epsilon}$, formula (55) yields an error in $\omega_{n}$ of order $\mathcal{O}(\epsilon)$, where the "big-oh" notation was used. Furthermore, due to the exponential term in formula (55), the approximate convolution integral in equation (54) may be efficiently computed using the FFT technique.

\section{NUMERICAL TESTS}

This section presents some numerical tests describing an in vivo ultrasound test on human cortical long bones. The cortical bone has a constant thickness $h=4 \mathrm{~mm}$. The bone is assumed to be a transversely isotropic medium whose tensor of elasticity is characterized by 5 independent constants $\left(c_{16}=c_{26}=0\right)$. Both fluid halfspaces $\left(\Omega_{1}^{f}\right.$ and $\left.\Omega_{2}^{f}\right)$ are assumed to be water.

The acoustic source (Eq. (2)) located at the position $\left(x_{1}^{s}, x_{2}^{s}\right)=(0,2)($ in $\mathrm{mm})$ in the upper fluid domain $\Omega_{1}^{f}$ has the time-history function given by:

$$
F(t)=F_{0} e^{-4\left(f_{c} t-1\right)^{2}} \sin \left(2 \pi f_{c} t\right),
$$

where $F_{0}=100 \mathrm{~m} . \mathrm{s}^{-2}$ and the central frequency $f_{c}=1 \mathrm{MHz}$. Fig. 2 depicts the time function of this signal $F(t)$ and its spectrum which contains a frequency bandwidth about $0-2.5 \mathrm{MHz}$ (obtained by Fourier transform). 

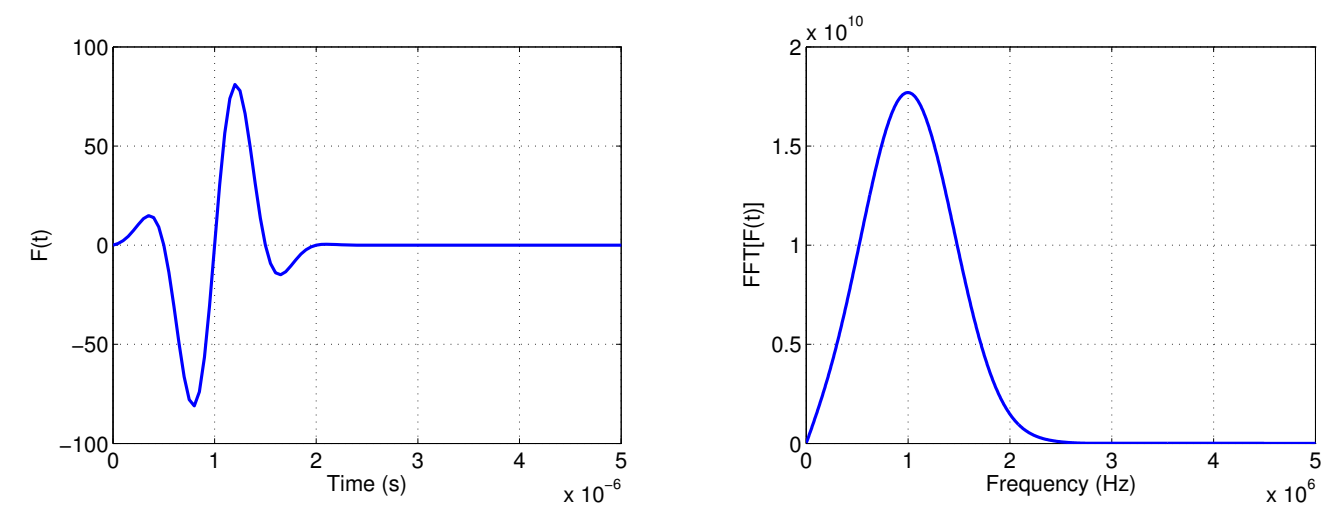

Fig. 2. Time-history function of the source (left) and its spectrum (right)

For all tests presented below, the total duration of the simulation will be taken by $T=2 \times 10^{-5} s$.

Two different cases are considered: (1) the first case considers the solid bone plate as a homogeneous medium; (2) the second case concerns a bone plate with a gradient of material properties, i.e. its physical characteristics are assumed to be homogeneous in the longitudinal direction on the $x_{1}$-axis but they vary along the depth on the $x_{2}$-axis.

\subsection{Case of a homogeneous solid bone - Validation}

Physical and numerical parameters. For this study, both fluid domains are assumed to be identical and their mechanical properties are given by $\rho_{1}=\rho_{2}=1000 \mathrm{kgm}^{-3}$ et $c_{1}=c_{2}=1500 \mathrm{~ms}^{-1}$. Table 1 provides the material parameters used for this test which correspond to the characteristics of a typical human compact bone [25]. According to these parameters presented, the phase velocities of compressional waves in $\Omega^{b}$ propagating along $x_{1}$ - and $x_{2}$-axes are $c_{p 1}=3698 \mathrm{~m} . \mathrm{s}^{-1}$ and $c_{p 2}=2984 \mathrm{~m} . \mathrm{s}^{-1}$, respectively.

Table 1. Mechanical properties of cortical bone obtained from [25]

\begin{tabular}{|c|c|c|c|c|c|}
\hline $\begin{array}{c}\rho \\
\left(\mathrm{kg} \cdot \mathrm{m}^{-3}\right)\end{array}$ & $\begin{array}{c}c_{11} \\
(\mathrm{GPa})\end{array}$ & $\begin{array}{c}c_{22} \\
(\mathrm{GPa})\end{array}$ & $\begin{array}{c}c_{12} \\
(\mathrm{GPa})\end{array}$ & $\begin{array}{c}c_{66} \\
(\mathrm{GPa})\end{array}$ & $\begin{array}{c}c_{16}=c_{26} \\
(\mathrm{GPa})\end{array}$ \\
\hline 1722 & 23.55 & 15.33 & 9.145 & 4.7 & 0 \\
\hline
\end{tabular}

The numerical parameters used for simulation have been chosen in a similar way to which required for a common procedure of dynamic finite element analysis (see e.g. [15]). The space interval $\Delta x$ is about the $1 / 10$ shortest wavelength. The time step is chosen by using the Courant-Friedrichs-Lewy condition. In order to obtain a bounded-value solution in space on the $\boldsymbol{e}_{1}$-axis, the domain size should be chosen sufficiently large to avoid the fact that fastest waves pass over the boundaries after the duration $T$.

For the finite element analysis on the $\boldsymbol{e}_{2}$-axis, the bone plate's thickness is discretized into 8 three-noded quadratic isoparametric Lagrangian elements. The numerical values of parameters required for the finite element analysis as well as for the inverse Laplace-Fourier transform are given in Table 2. 
Table 2. Parameters used for numerical simulation

\begin{tabular}{|c|c|c|c|c|c|c|}
\hline$n^{e l}$ & order & $\Delta x_{1}(\mu \mathrm{m})$ & $N_{1}$ & $T(\mu \mathrm{s})$ & $N_{t}$ & $\epsilon$ (Eq. 55) \\
\hline 8 & 3 & 75 & 2048 & 20 & 2048 & $10^{-12}$ \\
\hline
\end{tabular}

Results and validation. Fig. 3 presents snapshots of the wave field in the coupled trilayer system at different instants $t=2 \mu \mathrm{s}, t=4.5 \mu \mathrm{s}$, and $t=9.5 \mu \mathrm{s}$. At each point $\boldsymbol{x}$ in the domain, the snapshots presented in these graphs are $\log \left(\left|p_{1}(\boldsymbol{x}, t)\right|\right)$ if $\boldsymbol{x} \in \Omega_{1}^{f}$, $\log \left(\left|p_{2}(\boldsymbol{x}, t)\right|\right)$ if $\boldsymbol{x} \in \Omega_{2}^{f}$ and $\log \operatorname{vm}(\boldsymbol{x}, t)$ if $\boldsymbol{x} \in \Omega^{b}$ where $\operatorname{vm}(\boldsymbol{x}, t)$ denotes the Von Mises stress. In these graphs, we can clearly observe the transmission and reflection of ultrasonic waves through the interfaces. As the longitudinal elastic waves propagating in the solid are much faster than acoustic waves in the fluid, the wavefronts of two symmetrical head waves, which have truncated cone forms, are very well displayed in two last graphs in Fig. 3 . Note that using the proposed method allows us to take into account the exact radiation conditions of two fluid domains, therefore the results involve only the waves diffracted by the bone plate.
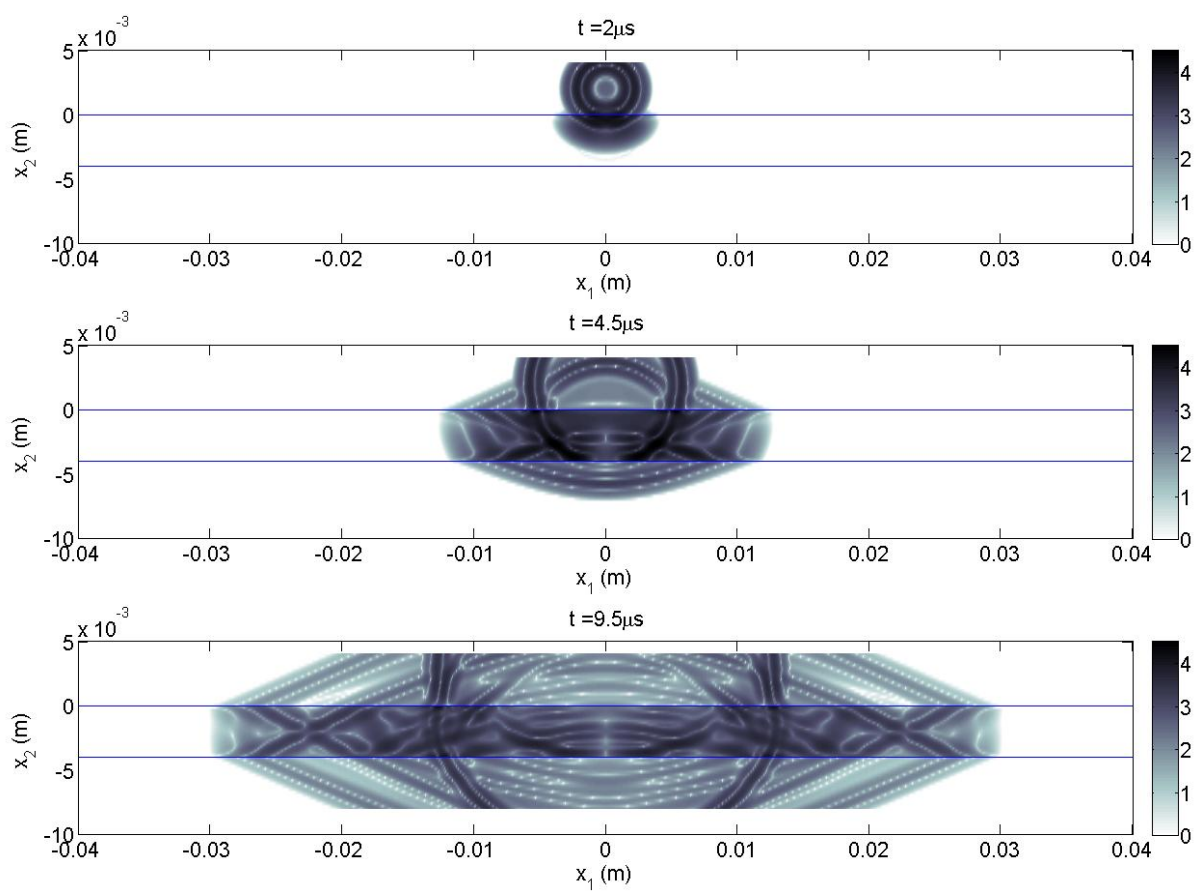

Fig. 3. Snapshot of waves at different instants

In Fig. 4, we depict the solutions of $p_{1}$ with respect to time $t$ at two receivers which are located at $R_{1}(2,2)$ (in $\mathrm{mm}$ ) and at $\mathrm{R}_{2}(20,2)$ (in $\mathrm{mm}$ ), respectively. At the receiver $\mathrm{R}_{1}$ that is close to the source, the first arrived perturbation is the direct wave, the second one is the wave reflected from the interface $\Gamma_{1}^{b f}$, and the last one is the contribution of the front wave (Fig. 4, upper). Otherwise, at the receiver $\mathrm{R}_{2}$ located at a distance further 
away from the source, the first arrived perturbation is the front wave, and the direct wave with larger amplitudes arrived later.
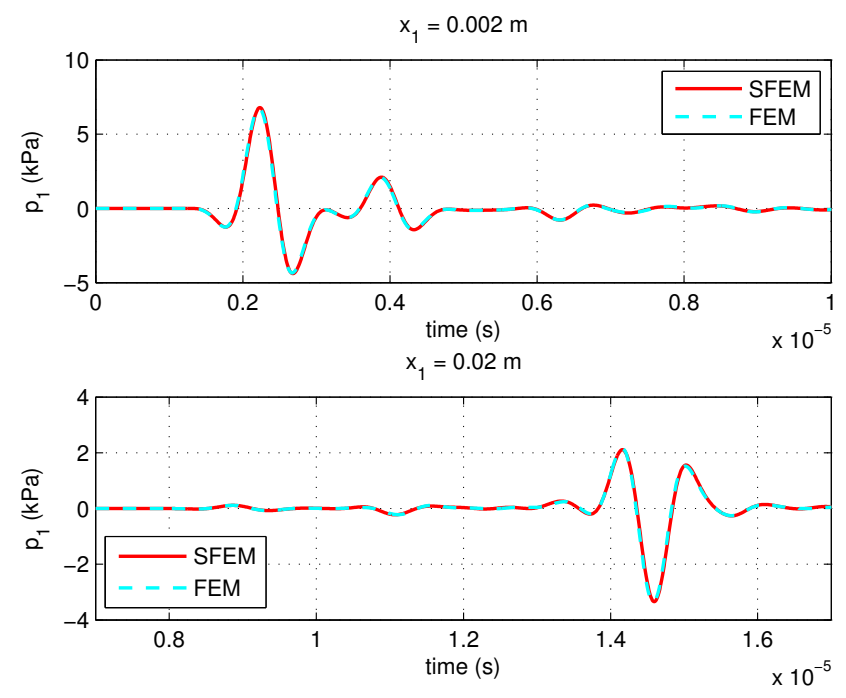

Fig. 4. Comparison between SFEM and FEM results: Acoustic pressures $p_{1}$ with respect to time at $\mathrm{R}_{1}(2,2)$ (in $\mathrm{mm}$ ) (upper) and at $\mathrm{R}_{2}(20,2)$ (in $\mathrm{mm}$ ) (lower)

For validation purposes, Fig. 4 also presents the results given by a classic time domain finite element analysis which were performed by using the software COMSOL Multiphysics. This shows that the results obtained by proposed SFE formulation are perfectly matched with the FE results. Fig. 5 presents SFE and FE solutions with respect to time $t$ of vertical displacement signals registered at two points $\mathrm{R}_{3}(2,-2)$ (in $\mathrm{mm}$ ) and $\mathrm{R}_{4}(20,-2)$ (in $\left.\mathrm{mm}\right)$. Again, the comparison shows good agreement between the results obtained by two methods.

While the classic dynamic finite element method needs about 4.5 hours for simulation, the proposed SFEM using a common laptop (Intel ${ }^{\circledR}$ Core ${ }^{\mathrm{TM}} 2 @ 3.06 \mathrm{GHz}$ ) needs only 1343 seconds to obtain the same results presented in Fig. 3. Moreover, for the tests realized during the study of the axial transmission technique, we essentially need only to get responses at the receivers. Hence, in practice, the computational time using SFEM is even smaller. For example, the total computational time required for the validations (Figs. 4 and 5 ) is only 277 seconds.

Convergence analysis. In order to perform a convergence analysis of the proposed method, we introduce a function $L_{2, T}^{p_{1}}(\boldsymbol{x})$ that is defined by:

$$
L_{2, T}^{p_{1}}(\boldsymbol{x})=\sqrt{\Delta t \sum_{n=0}^{N_{t}}\left(p_{1}(\boldsymbol{x}, n \Delta t)\right)^{2}},
$$



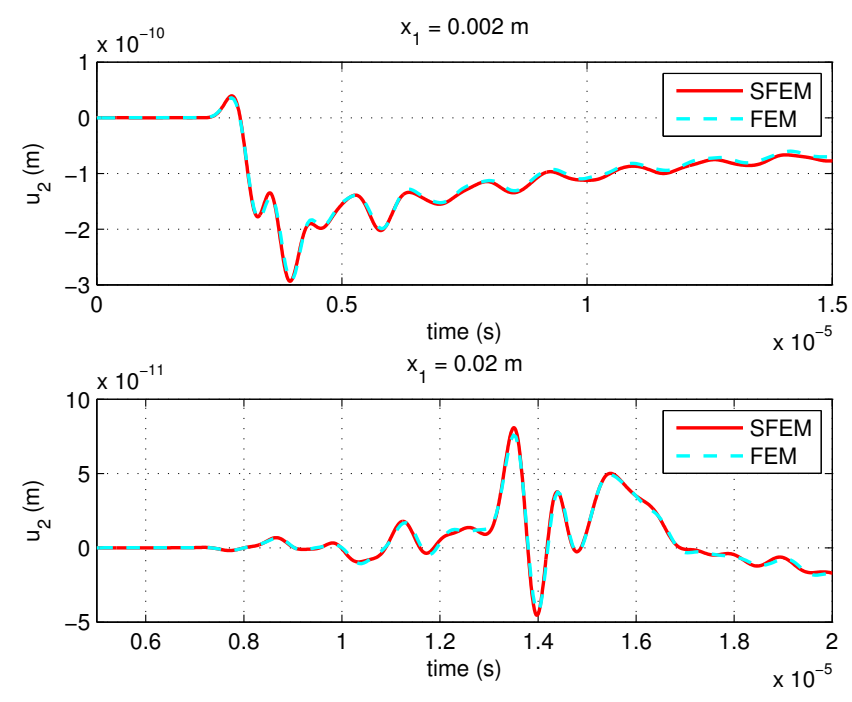

Fig. 5. Comparison between SFEM and FEM results: Vertical displacement $u_{2}$ with respect to time at $\mathrm{R}_{3}(2,-2)$ (in $\mathrm{mm}$ ) (upper) and at $\mathrm{R}_{4}(20,-2)$ (in $\mathrm{mm}$ ) (lower)

then the relative error at a point $\boldsymbol{x}$ is estimated by:

$$
\text { relative error }=\left|\frac{L_{2, T}^{p_{1}}(\boldsymbol{x})-L_{2, T}^{p_{1}, \mathrm{ref}}(\boldsymbol{x})}{L_{2, T}^{p_{1}, \mathrm{ref}}(\boldsymbol{x})}\right|,
$$

where $L_{2, T}^{p_{1}, \text { ref }}(\boldsymbol{x})$ is the reference value obtained from the FE analysis. Fig. 6 presents
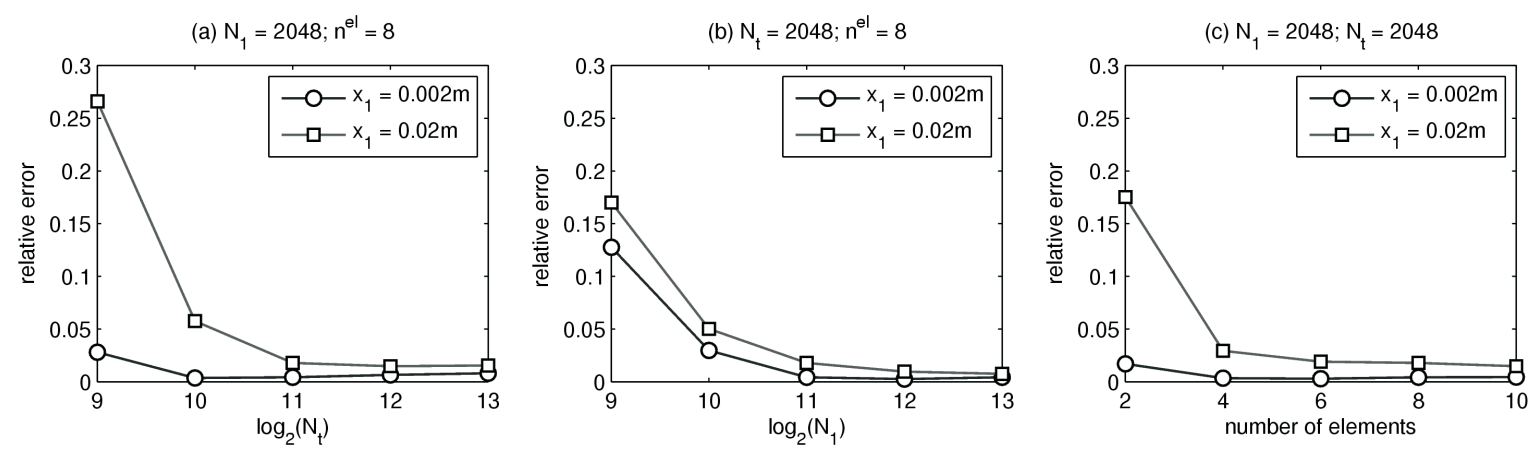

Fig. 6. Convergence studies: numerical errors versus (a) time sample number $\left(N_{t}\right)$, (b) space sample number $\left(N_{1}\right),(c)$ finite element discretization $\left(n^{e l}\right)$

convergence studies with respect to different parameters by calculating relative errors at two points $\mathrm{R}_{1}$ and $\mathrm{R}_{2}$. In Fig. $6 \mathrm{a}$, the parameters $N_{1}=2048$ and $n^{e l}=8$ which determine the discretization in space are fixed and the effect of the total number of time step $N_{t}$ (that is related to time step size $\Delta t$ ) on the convergence is shown. Next, Fig. 6b shows 
the effect of sampling number $N_{1}$, which is associated with the wavenumber $k_{1}$, by fixing $N_{t}=2048$ and $n^{e l}=8$. Last, Fig. $6 \mathrm{c}$ presents the relative errors versus the number of finite elements $n^{e l}$ when using $N_{1}=2048$ and $N_{t}=2048$. These convergence studies show that the proposed procedure using spectral finite elements has good stability and convergence.

\subsection{Case of a bone layer with functionally graded properties}

This case considers the same problem as in previous section but the bone solid layer is a graded-functionally medium. The motivation of this study arises from the fact that the porosity in the endosteal zone (inner part of the bone) is significantly more important than in the periosteal zone (outer part of the bone) [26]. As a consequence, the mass density and rigidity of cortical bone are heterogeneous in the radial direction.

Table 3. Variation of elastic properties in the solid bone layer

\begin{tabular}{|l|c|c|c|c|c|}
\hline Material properties & $\begin{array}{c}\rho_{s} \\
\left(\mathrm{~kg} . \mathrm{m}^{-3}\right)\end{array}$ & $\begin{array}{c}c_{11} \\
(\mathrm{GPa})\end{array}$ & $\begin{array}{c}c_{22} \\
(\mathrm{GPa})\end{array}$ & $\begin{array}{c}c_{12} \\
(\mathrm{GPa})\end{array}$ & $\begin{array}{c}c_{66} \\
(\mathrm{GPa})\end{array}$ \\
\hline Min. value at $x_{2}=-h$ & 1660 & 17.6 & 11.8 & 5.1 & 3.3 \\
\hline Max. value at $x_{2}=0$ & 1753 & 29.6 & 25.9 & 11.1 & 5.5 \\
\hline
\end{tabular}

For this test, the mass density and coefficients of the elasticity tensor are assumed to vary linearly with respect to the depth on the $x_{2}$-axis and obtain minimum values at the lower surface $\left(x_{2}=-h\right)$. Table 3 gives the set of the minimum and maximum values of these properties which correspond to a typical cortical bone [11, 25].

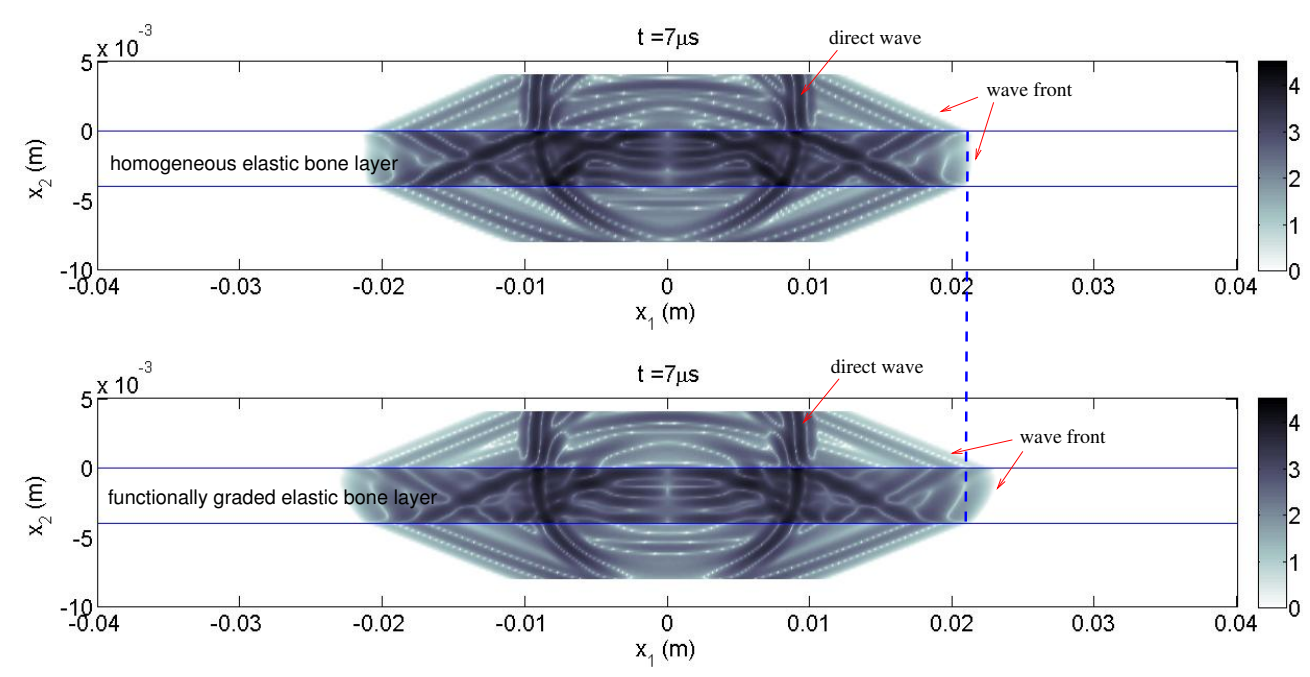

Fig. \%. Effect of graded functionally properties of the bone layer

In order to display the quantitative effect of the material properties of the gradient, we present in Fig. 7 two typical snapshots of the wave field captured at $t=7 \mu \mathrm{s}$ in cases 
of homogeneous and heterogeneous bones, respectively. For the homogeneous layer (Fig. 7 , upper), the profile of the fastest wavefront is nearly perpendicular to the bone surface, whereas for the heterogeneous bone layer (see Fig. 7, lower), the profile of wavefront has a significant slope. This inclination angle of wavefront profile reflects well the fact that the wave velocities in bone plate decreases according to the position in depth on the $x_{2}$-axis of the considered point.

\section{CONCLUSION}

Mechanical modeling of QUS using axial transmission technique deals with a timedomain vibro-acoustic problem. This configuration may be modeled by a two-dimensional trilayer medium which consists of a solid layer (representing cortical bone layer) sandwiched between two fluid media (representing soft tissues such as skin or marrow). The bone material is assumed to be an anisotropic elastic solid and the fluids are idealized as two acoustic fluid halfspaces.

The proposed spectral finite element formulation has been shown to be not only very stable for the simulation of the propagation of ultrasonic waves through a functionally graded anisotropic elastic bone plate immersed in a fluid, but also very competitive in computational time. Some advantages of this method may be outlined. Firstly, in the Laplace-Fourier domain, the presence of two halfspace domains may be represented by exact radiation conditions derived from the analytical solutions of the waves in the fluids. Consequently, the finite element modeling may be performed uniquely for the solid domain. Secondly, due to the one-dimension problem constructed in $\left(s-k_{1}\right)$ domain, the memory required for computation is very small in comparison with that required by two-dimensional finite element analysis in the time-domain. In comparison with the spectral finite element obtained which applies a Fourier transform on a time variable, using the Laplace transform allows us to avoid well-known numerical difficulties concerning the singularities at the real frequency poles. Moreover, it has been shown that the Convolution Quadrature Method is an efficient and stable technique in order to evaluate the time-domain solutions for the transient high-frequency problem presented here.

The proposed method allows us to consider the anisotropy as well as the heterogeneity of the solid material. The numerical results have shown that the spatial gradient of material properties with depth has a remarkable influence on the behavior of ultrasonic waves propagating in cortical bones.

Although the method presented in this paper is used for ultrasonic diagnostic of cortical bones, it can be applied for other fields in which the configuration may be an unbounded or bounded structure with an arbitrary continuous or discontinuous profile of mechanical properties in depth. Moreover, the proposed formulation has the potential to take into account the effect of viscoelasticity with or without memory in materials.

\section{REFERENCES}

[1] G. Lowet, G. Van der Perre, Ultrasound velocity measurements in long bones: measurement method and simulation of ultrasound wave propagation, Journal of Biomechanics, 29 (1996), $1255-1262$. 
[2] P. Nicholson, P. Moilanen, T. Karkkainen, J. Timonen and S. Cheng, Guided ultrasonic waves in long bones: modelling, experiment and in vivo application, Physiological Measurement, 23(4) (2002), 755 - 768.

[3] P. Moilanen, Ultrasonic guided waves in bone, IEEE Transactions of Ultrasonics, Ferroelectrics, and Frequency Control, 55(6) (2008), 1277 - 1286.

[4] H. A. Nayfeh, Wave Propagation in Layered Anisotropic Media, Elsevier, (1995).

[5] C. Baron, S. Naili, Elastic wave propagation in a fluid-loaded anisotropic waveguide with laterally varying properties, C.R. Mécanique, 336 (2008), 772 - 730.

[6] C. Baron, S. Naili, Propagation of elastic wavesin a fluid-loaded anisotropic functionally graded waveguide: Application to ultrasound characterization, J. Acoust. Soc. Am, 127(3) (2010), $1307-1317$.

[7] A. Shuvalov, O. Poncelet and M. Deschamps, Analysis of the dispersion spectrum of fluidloaded anisotropic plates: leaky-wave branches, Journal of Sound and Vibration, 296(3) (2006), $494-517$.

[8] E. Bossy, M. Talmant, P. Laugier, Effect of bone cortical thickness on velocity measurements using ultrasonic axial transmission: A 2D simulation study, J. Acoust. Soc. Am., 112 (2002), $297-307$.

[9] Q. Grimal, S. Naili, A theoretical analysis in the time-domain of wave reflection on a bone plate, J. Sound Vib., 298 (2006), 12 - 29.

[10] C. Desceliers, C. Soize, Q. Grimal, G. Haiat and S. Naili, A time-domain method to solve transient elastic wave propagation in a multilayer medium with a hybrid spectral-finite element space approximation, Wave Motion, 45(4) (2008), 383 - 399.

[11] G. Häat, S. Naili, Q. Grimal, M. Talmant, C. Desceliers and C. Soize, Influence of a gradient of material properties on ultrasonic wave propagation in cortical bone: Application to axial transmission, J. Acoust. Soc. Am., 125(6) (2009), 4043 - 4052.

[12] E. Kausel, Fundamental Solution in Elastodynamics, Cambridge University Press, (2006).

[13] N. A. Kampanis, V. A. Dougalis, and J. A. Ekaterinaris (Eds), Effective Computational Methods for Wave Propagation, Chapman \& Hall/CRC, (2008).

[14] J. Virieux, P-SV-wave propagation in heterogeneous media: velocity-stress finite-difference method, Geophysics, 51(4) (1986), 889 - 901.

[15] K. J. Bathe, Finite Element Procedure, Prentice Hall, (1996).

[16] D. Givoli, High-order local non-reflecting boundary conditions: a review, Wave Motion, 39(4) (2004), $319-326$.

[17] L. L. Thompson, R. Huan, Implementation of exact non-reflecting boundary conditions in the finite element method for the time-dependent wave equation, Computer Methods in Applied Mechanics and Engineering, 187(1-2) (2000), 137 - 159.

[18] X. Han, G. R. Liu, and K. Y. Lam, Transient waves in plates of functionally graded materials, journal = International Journal for Numerical Methods in Engineering, 52(8) (2001), 851 865.

[19] X. Han, G. R. Liu, Z. C. Xi, and K. Y. Lam, Transient waves in a functionally graded cylinder, International Journal of Solids and Structures, 38(17) (2001), 3021 - 3037.

[20] G. R. Liu, Z. C. Xi, Elastic Waves in Anisotropic Laminates, CRC Press, (2002).

[21] S. Gopalakrishnan, A. Chakraborty, and D. Roy Mahapatra, Spectral Finite Element Method, Spinger, (2008).

[22] A. Marzani, Time-transient response for ultrasonic guided waves propagating in damped cylinders, International Journal of Solids and Structures, 45(25-26) (2008), 6347 - 6368. 
[23] J. Carcione, Wave Fields in Real Media: Wave Propagation in Anisotropic, Anelastic and Porous Media, Elsevier, (2001).

[24] M. Schanz, H. Antes, Application of 'Operational Quadrature Methods' in Time Domain Boundary Element Methods, Meccanica, 32 (1997), 179 - 186.

[25] N. X. Dong, E. X. Guo, The dependence of transverse isotropic elasticity of human femoral cortical boneon porosity, J. Biomechanics, 37(8) (2004), 1281 - 1287.

[26] C. Thomas, S. A. Feik, and J. Clement, Regional variation of intracortical porosity in the midshaft of the human femur: age and sex differences, J. Anat., 206 (2005), 115 - 125.

Received July 6, 2011 